\title{
Parametric estimation of the immunes proportion based on BCH model and exponential distribution using left censored data.
}

\begin{abstract}
In population based cancer clinical trials, a proportion of patients will never experience the interested event and considered as "cured" or "immunes". The majority of recent cancer studies focus on the estimation of immune proportion. In this study we investigated the estimation of proportion of patients curd of cancer in case of left censored data based on the Bounded Cumulative Hazard (BCH) model proposed by Chen in 1999. The analysis provided the Maximum Likelihood Estimation (MLE) of the parameters within the framework of the Expectation Maximization (EM) algorithm where the numerical solutions of the estimation equations of the cure rate parameter could be employed.
\end{abstract}

Keyword: Cure fraction; BCH model; Left censoring; MLE method; EM algorithm. 